\title{
Equidad en educación superior: experiencia de un programa especial de ingreso a carreras de la salud
}

\author{
VERÓNICA GAETE ${ }^{(1)}$, FABIO SÁENZ ${ }^{(2)}$, RODRIGO DE LA FABIÁN ${ }^{(3)}$, JORGE LAS HERAS ${ }^{(4)}$, GLORIA RIQUELME ${ }^{(5)}$,
} JOSÉ NAVARRO $^{(6)}$ y CAROLINA LÓPEZ ${ }^{(7)}$

\section{RESUMEN}

Propósito: Describir los resultados de los primeros 5 años de un programa de ingreso diferenciado de estudiantes destacados de nivel socioeconómico bajo a carreras de la salud de la Facultad de Medicina de la Universidad de Chile. Material y Método: Se incorporaron anualmente 10 a 20 alumnos de $3^{\circ}$ y $4^{\circ}$ Medio de escasos recursos por sus antecedentes académicos y psicosociales. Durante uno o dos años recibieron refuerzo en ramos científicos y habilidades para la vida, tutoría y orientación vocacional.Rindieron también la PAA/PSU. De los preseleccionados, se eligieron hasta 5 por año para ingresar a la Facultad de acuerdo a sus antecedentes académicos, vocacionales y condiciones psicosociales de adaptación. Cursaron las carreras con beca completa y otros apoyos económicos. A aquellos que no ingresaron se les facilitaron otras instancias de educación superior. Resultados: A la fecha, han participado 57 estudiantes, 43,9\% mujeres y 56,1\% hombres. Su promedio de notas de enseñanza media fue $x$ $=6,1.43,5 \%$ no tenía expectativas previas de ingresar a la universidad. Durante el apoyo inicial 41,1\% evidenció déficit de hábitos y técnicas de estudio, 67,3\% desorientación vocacional, 33,3\% psicopatología y 38,6\% conductas de riesgo. 15,8\% desertó y 17,5\% fue excluido. La deserción se debió principalmente a problemas vocacionales y stress, y la exclusión a mal rendimiento y psicopatología. 47,4\% de los que finalizaron la etapa previa fue seleccionado para cursar carreras en la Facultad. Sus puntajes ponderados estuvieron bajo los mínimos para el ingreso regular. Su desempeño posterior fue en general satisfactorio. Conclusiones: El programa generó oportunidades de acceso a la universidad fuera de las expectativas de este grupo de jóvenes. Su realidad psicosocial dificultó su desempeño. El apoyo en esta área es muy importante en este tipo de programas. Su rendimiento en la Facultad fue satisfactorio a pesar de que no habrían sido admitidos por el proceso regular. Para generar real equidad en la educación universitaria es necesario ampliar los criterios de selección para estudiantes de escasos recursos.

Palabras clave: Evaluación de programas y proyectos de salud, Educación, Criterios de admisión escolar, Apoyo a la formación profesional, Estudiantes del área de la salud, Pobreza, Población de bajos salarios.

\footnotetext{
${ }^{(1)}$ Centro de Salud del Adolescente SERJOVEN. Departamento de Pediatría y Cirugía Infantil Campus Oriente. Facultad de Medicina. Universidad de Chile. Av. Raúl Labbé º 13.649. Lo Barnechea. Santiago. Chile. mgaete@med.uchile.cl

(2) Psicólogo. Centro de Salud del Adolescente SERJOVEN. Ayudante. Departamento de Pediatría y Cirugía Infantil Oriente. Facultad de Medicina. Universidad de Chile. Chile.

(3) Psicólogo. Magíster en Psicopatología Fundamental y Psicoanálisis. Centro de Salud del Adolescente SERJOVEN. Chile.

(4) Médico Cirujano. Doctor en Patología. Profesor Titular, Prorrector Universidad de Chile. Chile.

${ }^{(5)}$ Licenciada en Física. Doctora en Ciencias. Profesor Titular Instituto de Ciencias Biomédicas. Facultad de Medicina. Universidad de Chile. Chile.

(6) Profesor de Biología y Ciencias. Magíster en Biología. Director Programa Beca Diego Peralta. Profesor Asociado. Instituto de Ciencias Biomédicas. Facultad de Medicina. Universidad de Chile. Chile.

(7) Psicóloga. Centro de Salud del Adolescente SERJOVEN. Ayudante Departamento de Pediatría y Cirugía Infantil Oriente. Facultad de Medicina. Universidad de Chile. Chile.
} 


\section{ABSTRACT \\ EQUITY IN HIGHER EDUCATION: EXPERIENCE FROM A SPECIAL PROGRAM FOR ADMISSION TO HEALTH CAREERS}

Purpose: To describe the results from the first 5 years of a differentiated admission program designed for outstanding students of low socioeconomic income admitted to health careers of the Faculty of Medicine, University of Chile. Materials and Methods: Annually, 10 to 20 low-incomestudents from 3rd and 4th years of secondary school entered the program in virtue of their academic and psychosocial background. For one or two years, they received extra support in scientific subjects and abilities for life, tutoring and vocational guidance. They also took the PAA/PUU (general post-secondary school test). Of the students who succeeded the pre-selection, up to 5 per year were chosen for admission to University according to their academic and vocational background and their psychosocial adaptation conditions. They studied their careers under full bursary coverage and other financial supports. Those who failed admission were helped to access other instances of higher education. Results: To date, 57 students have participated in the program. Of these, 43,9\% are females and 56,1\% are men. Their average secondary school grade was $x$ $=6,1$. In total, 43,5\% had no prior expectations on being admitted to university. During the initial support delivery, 41,1\% evidenced a lack of study habits and techniques, 33,3\% showed a psychopathological condition and 38,6\% evidenced risk behaviors. In 15,8\% of the cases students dropped out and in 17,5\% there was student exclusion. Drop out was due mainly to vocational problems and stress, and exclusion was due to low performance and psychopathological conditions. Of the students who completed the previous stage, 47,4\% was selected to study careers at the Faculty. Their test scores were below the minimum for regular admission. In general, their subsequent performance was satisfactory. Conclusions: The program generated university access opportunities that were beyond the expectations of this group of youngsters. Their psychosocial facts hindered their performance. The support in this area is very significant for this kind of program. Students' performance within the Faculty was satisfactory in spite of the fact they wouldn't have been admitted through the regular admission process. A widening of selection criteria for low-income students is necessary to generate equity in university education.

Keywords: Program evaluation; Training programs; School admission criteria; Educational subsidies; Health occupations students; Low income population).

\section{INTRODUCCIÓN}

La educación, a través del desarrollo del capital humano, tiene un gran impacto en el bienestar socioeconómico en las sociedades contemporáneas ${ }^{1}$, constituyendo una de las variables más importantes en la generación de equidad social. En ella recae, en gran medida, la posibilidad de romper el círculo de la pobreza, caracterizado por la tendencia a la reproducción transgeneracional de hogares carenciados en lo económico y social ${ }^{2}$.
En el ámbito de la educación, el acceso a estudios superiores es el que tiene mayor incidencia en los aspectos previos. Cálculos recientes revelan que mientras cada año adicional de educación básica y media incrementa la renta en $4 \%$ y $10 \%$ respectivamente, cada año de educación superior lo hace en $20 \%^{3}$.

Chile se ubica entre los países de América Latina y El Caribe con mayor cobertura de enseñanza superior ${ }^{4}$. Sin embargo, resultan evidentes los problemas de equidad en el acceso 
a ella. La encuesta CASEN 2003 reveló que la cobertura creció $21,5 \%$ desde 1990 , pero también que la brecha entre los tres quintiles más pobres y los dos de mayores ingresos ha aumentado ${ }^{5,6}$.

Los estudiantes chilenos de escasos recursos no sólo presentan desventajas en el acceso a la educación superior. Resulta esperable que las carencias económicas, educativas, psicológicas y sociales entre las que han crecido, aumenten también sus posibilidades de fracaso.

Lo anterior, en el contexto de un país que presenta una de las mayores desigualdades en la distribución del ingreso de Latinoamérica y el mundo ${ }^{7}$, permite inferir que para generar equidad social en Chile, resulta esencial mejorar tanto el acceso a la educación superior de nuestros jóvenes de escasos recursos, como sus posibilidades permanecer y tener éxito en ésta.

Las estrategias implementadas -tanto gubernamentales como de otras institucioneshan resultado insuficientes en este sentido, tendiendo a centrarse en brindar apoyo económico a estudiantes de escasos recursos que cumplen con todos los requisitos tradicionales de selección universitaria ${ }^{8,9}$.

El programa que se presenta a continuaciónBeca Diego Peralta-, pretende ser un aporte en este ámbito. Fue gestado por la Facultad de Medicina de la Universidad de Chile, para intentar revertir la preocupante tendencia a la disminución del ingreso de jóvenes de escasos recursos a la misma. Se realiza en colaboración con las municipalidades de las comunas involucradas y la familia del trágicamente fallecido alumno de Medicina del cual tomó su nombre. Su objetivo es brindar a estudiantes destacados en situación de pobreza y alto riesgo social, la posibilidad de estudiar con éxito una de las ocho carreras de la Facultad de Medicina, a través de un acceso diferenciado y una intervención precoz e integral, que contempla no sólo apoyo económico, sino además académico, psicológico y social.

El objetivo general de este estudio es sistematizar y analizar descriptivamente los resultados del Programa en sus primeros 5 años. Sus objetivos específicos son:
1. Describir algunas características académicas, psicológicas, sociales y familiares de los participantes.

2. Describir su evolución académica y psicosocial durante el proceso de apoyo previo al ingreso a la Facultad y el desempeño académico inicial de aquellos que ingresaron a las distintas carreras.

\section{MATERIAL Y MÉTODO}

El Programa se inició el 2001. A él se incorporan anualmente 10 a 20 alumnos destacados de $3^{\circ}$ y $4^{\circ}$ Medio de colegios municipalizados y particulares subvencionados de comunas de alto riesgo social de Santiago (Cerro Navia y La Pintana). Éstos se seleccionaron considerando requisitos académicos y psicosociales. Entre los primeros, su rendimiento escolar, un coeficiente intelectual (C.I.) acorde a estudios universitarios y sus conocimientos y habilidades en matemáticas, ciencias (física, química y biología) y comprensión de lectura. Entre los segundos, un perfil vocacional acorde con carreras del área de la salud, ausencia tanto de psicopatología incompatible con el programa (abuso/dependencia de alcohol o drogas, trastorno de personalidad, trastorno psicótico y/o trastorno orgánico-cerebral), como de conductas reñidas con la ética y problemas legales, y existencia de apoyo familiar. Para determinar dichas variables se utilizaron el promedio de notas de enseñanza media, el Test de Matrices Progresivas de Raven ${ }^{10}$, pruebas escritas de matemáticas, ciencias y comprensión de lectura, el Inventario de Preferencias Personales de Holland ${ }^{11}$, el Inventario Multifásico de Personalidad de Minnesota $(\mathrm{MMPI})^{12}$ y entrevistas psicológica y social.

Los participantes se incorporaron inicialmente a un proceso de apoyo integral por un equipo multidisciplinario de la Facultad de Medicina, cuya duración varió entre uno y dos años dependiendo si al ingresar cursaban $3^{\circ} \mathrm{o}$ $4^{\circ}$ Medio. Durante este período, además de su educación regular, los alumnos recibieron refuerzo en biología, química, física y 
matemáticas. Junto con esto, realizaron un taller en el que se facilitó su entrenamiento en habilidades para la vida, tales como autoestima, comunicación asertiva, resolución de conflictos, pensamiento crítico y manejo de la ansiedad y frustración. También asistieron regularmente a sesiones de tutoría individual y recibieron orientación vocacional. Paralelamente, se brindó atención psicológica y social a quienes las requirieron. Las actividades previas fueron evaluadas mediante pruebas escritas en las asignaturas reforzadas y una encuesta de percepción del impacto del taller. Durante esta etapa se realizaron evaluaciones semestrales del desempeño global de los estudiantes, excluyéndose aquellos que cursaron con mal rendimiento, presentaron problemas legales o éticos y/o evidenciaron la psicopatología ya descrita. Además, los alumnos rindieron la prueba nacional de selección universitaria (Prueba de Aptitud Académica -PAA- hasta el 2002 o Prueba de Selección Universitaria -PSUa partir del 2003).

Al término del proceso previo se seleccionaron hasta 5 alumnos por año para ingresar a las carreras de la Facultad de Medicina. Para tal efecto, se consideraron su promedio de notas de enseñanza media, su rendimiento académico en las materias reforzadas $(\geq 5,0)$, sus puntajes promedio PAA/PSU (Verbal/Lenguaje y Comunicación, Matemáticas y Biología/Ciencias), su asistencia al Programa ( $\geq 85 \%)$ y sus preferencias vocacionales. Esto se enmarcó dentro de la normativa de admisión especial de la Universidad de Chile. Los escogidos se incorporaron a los programas regulares de las carreras, las que cursaron sin costo, y recibieron -además- una beca de alimentación y dinero para movilización y textos. Como alumnos regulares tuvieron también acceso a apoyo psicosocial en el Programa de Tutoría de Alumnos. Su desempeño fue evaluado de manera idéntica que el del resto del alumnado de la Facultad.

Con el propósito de que el Programa generara oportunidades de desarrollo positivo para todos los participantes, a aquellos que no ingresaron a la Facultad se les facilitó -mediante información y contactos- el acceso a otras instancias de educación superior, idealmente de su vocación y subvencionadas.

El Programa incluyó actividades adicionales para los colegios participantes: capacitación en biología para profesores y charlas de salud para la comunidad escolar.

\section{RESULTADOS}

A continuación se presentan los resultados correspondientes a la totalidad de alumnos ingresados al Programa en el período 20012005, exceptuando aquellos que recién cursaban $3^{\circ}$ Medio el 2005, por considerarse insuficiente su tiempo de participación para este análisis.

Se trató de 57 estudiantes, 43,9\% mujeres y $56,1 \%$ hombres, cuya edad promedio al ingreso fue 17,1 años (rango: 15,8 a 20,5 años). Se consigna entre paréntesis aquellos casos en que el total es menor.

\section{a. Características académicas, psicológicas, sociales y familiares de los participantes.}

El $53,5 \%$ de los alumnos iniciaba $3^{\circ}$ Medio y el $46,5 \% 4^{\circ}$ medio. Si bien el 93,5\% (43/46) había proyectado cursar educación superior, el $39,5 \%$ pensaba realizarla en institutos técnicoprofesionales. En total, el 43,5\% (20/46) no tenia expectativas previas de ingresar a la universidad.

Su promedio de notas de enseñanza media fue 6,1 (rango: 5,6 a 7,0). El 60,9\% (28/46)

Tabla 1. Estructura familiar alumnos beca Diego Peralta Facultad de Medicina U. de Chile

\begin{tabular}{|l|c|}
\hline \multicolumn{1}{|c|}{ Tipo Familia } & $\begin{array}{c}\mathbf{\%} \\
\mathbf{( N = 5 5 )}\end{array}$ \\
\hline Biparental (nuclear o extensa) & 63,6 \\
\hline Nuclear monoparental & 12,7 \\
\hline Extensa compuesta & 12,7 \\
\hline Simultánea & 1,8 \\
\hline Otra & 9,2 \\
\hline
\end{tabular}


Tabla 2. Nivel educacional de los padres alumnos beca Diego Peralta Facultad de Medicina U. de Chile

\begin{tabular}{|l|c|c|}
\hline \multicolumn{1}{|c|}{ Nivel educacional } & $\begin{array}{c}\text { Madres o } \\
\text { sustitutas } \\
\mathbf{( N = 5 7 )}\end{array}$ & $\begin{array}{c}\text { Padres o } \\
\text { sustitutos } \\
(\mathbf{N = 5 4 )}\end{array}$ \\
\hline Analfabeto(a) & - & $1,9 \%$ \\
\hline Educación primaria incompleta & $19,3 \%$ & $18,5 \%$ \\
\hline Educación primaria completa & $47,4 \%$ & $37,0 \%$ \\
\hline Educación secundaria completa & $29,8 \%$ & $38,9 \%$ \\
\hline Educación Universitaria & $3,5 \%$ & $3,7 \%$ \\
\hline
\end{tabular}

presentó un C.I. ubicado en el rango Superior Término Medio, el 28,3\% en el Superior y el $10,9 \%$ en el Termino Medio. Su perfil vocacional resultó compatible con carreras del área de la salud en el $36,2 \%$ (17/47), poco definido en el $34,0 \%$, dudoso en el $19,1 \%$ e incompatible en el $10,6 \%$. En cuanto a indicios de psicopatología, el MMPI evidenció que el 64,4\% (29/45) no los presentaba, el $28,9 \%$ presentaba entre 1 y 2 escalas desviadas y el 6,7\% entre 3 y 5 .

La estructura familiar y el nivel educacional de los padres de los participantes se presentan en las tablas 1 y 2 . Si bien el $87,2 \%$ de los padres y madres (34/39) proyectaba para sus hijos la educación superior, el 52,9\% esperaba que cursaran la técnico-profesional y sólo un 47,1\% la universitaria. En total, el 59,0\% no esperaba que sus hijos ingresasen a la universidad previo al Programa.

\section{b. Evolución académica y psicosocial de los participantes durante el proceso de apoyo previo al ingreso a la Facultad y desempeño académico inicial de aquellos que ingresaron a las distintas carreras.}

En el período de apoyo previo al ingreso a la Facultad, el 41,1\% (23/56) de los alumnos presentó déficit de hábitos y técnicas de estudio. Junto con esto, el 67,3\% (37/55) cursó con desorientación vocacional y el 33,3\% evidenció psicopatología. Esta última correspondió en $60,0 \%$ de los casos $(12 / 20)$ a un trastorno adaptativo y en $10,0 \%$ a trastornos depresivo, somatomorfo, de ansiedad y de la personalidad, respectivamente. El 38,6\% presentó también conductas o condiciones de alto riesgo, siendo las más frecuentes la actividad sexual no protegida $(47,8 \%)$ y la intención de iniciar relaciones coitales $(39,1 \%)$. El consumo de sustancias, las conductas violentas y la ideación suicida representaron el $4,4 \%$ del total, respectivamente.

La participación en el taller de habilidades para la vida generó la percepción de aumento de la asertividad en el $100 \%(33 / 33)$, de la capacidad para manejar la ansiedad y frustración en el 96,9\%, del espíritu crítico en el 93,9\%, de la autoestima en el $84,8 \%$ y de las habilidades para resolver conflictos en el 92,3\% (24/26).

Sus familias se comportaron como funcionales en el $56,1 \%$ de los casos, leve o

Tabla 3. Causas de deserción del programa beca Diego Peralta en la etapa de apoyo previa al ingreso a la Facultad de Medicina U. de Chile

\begin{tabular}{|l|c|}
\hline \multicolumn{1}{|c|}{ Causas deserción } & $\begin{array}{c}\mathbf{N}^{\text {o }} \\
\text { Alumnos }\end{array}$ \\
\hline Ausencia de vocación por el área de la salud & 2 \\
\hline Stress & 2 \\
\hline "Deseo de dedicarse exclusivamente al colegio" & 2 \\
\hline Embarazo de la pareja & 1 \\
\hline Patología oftalmológica invalidante & 1 \\
\hline $\begin{array}{l}\text { Ausencia de vocación por el área } \\
\text { de la salud + stress }\end{array}$ & 1 \\
\hline TOTAL & 9 \\
\hline
\end{tabular}

Tabla 4. Causas de exclusión del programa beca Diego Peralta en la etapa de apoyo previa al ingreso a la Facultad de Medicina U. de Chile

\begin{tabular}{|l|c|}
\hline \multicolumn{1}{|c|}{ Causas exclusión } & $\begin{array}{c}\mathbf{N}^{\mathbf{0}} \\
\text { Alumnos }\end{array}$ \\
\hline Mal rendimiento académico & 6 \\
\hline $\begin{array}{l}\text { Psicopatología } \\
\text { (trastorno de personalidad grave) }\end{array}$ & 2 \\
\hline $\begin{array}{l}\text { Mal rendimiento académico + } \\
\text { Psicopatología (t. de personalidad grave) }\end{array}$ & 1 \\
\hline Conductas antisociales & 1 \\
\hline TOTAL & 10 \\
\hline
\end{tabular}


Tabla 5. Diferencia entre puntajes ponderados de ingreso alumnos beca Diego Peralta y estudiantes regulares de las carreras Facultad de Medicina U. de Chile

\begin{tabular}{|c|l|c|c|c|}
\hline $\begin{array}{c}\mathrm{N}^{\mathrm{N}} \\
\text { Alum. }\end{array}$ & $\begin{array}{l}\text { Carrera } \\
\text { ingreso }\end{array}$ & $\begin{array}{c}\text { Puntaje } \\
\text { ponderado }\end{array}$ & $\begin{array}{c}\text { Puntaje } \\
\text { último } \\
\text { matriculado }\end{array}$ & $\begin{array}{c}\text { Dif. } \\
\text { (puntos) }\end{array}$ \\
\hline 1 & T. Ocupacional & 511 & 674 & -163 \\
\hline 2 & Medicina & 574 & 749 & -175 \\
\hline 3 & Medicina & 585 & 749 & -164 \\
\hline 4 & Medicina & 589 & 753 & -164 \\
\hline 5 & Medicina & 693 & 752 & -59 \\
\hline 6 & Enfermería & 575 & 667 & -92 \\
\hline 7 & Enfermería & 591 & 637 & -46 \\
\hline 8 & Enfermería & 599 & 657 & -58 \\
\hline 9 & Fonoaudiología & 649 & 678 & -29 \\
\hline 10 & Fonoaudiología & 560 & 682 & -122 \\
\hline 11 & Fonoaudiología & 569 & 680 & -111 \\
\hline 12 & Kinesiología & 627 & 711 & -84 \\
\hline 13 & Kinesiología & 455 & 725 & -270 \\
\hline 14 & Obstetricia & 602 & 629 & -27 \\
\hline 15 & Obstetricia & 493 & 629 & -136 \\
\hline 16 & Tecnología Médica & 539 & 683 & -144 \\
\hline 17 & Tecnología Médica & 591 & 683 & -92 \\
\hline 18 & Tecnología Médica & 649 & 689 & -40 \\
\hline & PROMEDIO & 580,6 & 690,4 & $-109,8$ \\
\hline & & & \\
\hline
\end{tabular}

moderadamente disfuncionales en el $36,8 \%$ y severamente disfuncionales en el $7,0 \%$.

El $66,7 \%$ de los participantes completó el proceso de apoyo previo al ingreso a la Facultad, mientras que el 15,8\% desertó y el 17,5\% fue excluido. Las causas de estas últimas se detallan en las tablas 3 y 4 . Aquellos que lo completaron obtuvieron un puntaje promedio PAA/PSU de 514,9 en Verbal/Lenguaje y Comunicación (rango: 283 a 693), de 554,9 en Matemáticas (rango: 368 a 713) y de 516,9 en Biología/Ciencias (rango: 352 a 669$)$.

El 47,4\% (18/38) de los alumnos que finalizaron la etapa previa fue seleccionado para ingresar a la Facultad ( 10 hombres y 8 mujeres). Éstos obtuvieron un puntaje promedio PAA/PSU de 550,1 en Verbal/Lenguaje y Comunicación (rango: 378 a 693), de 590,3 en Matemáticas (rango: 438 a 713) y de 541,9 en Biología/Ciencias (rango: 398 a 669). 4 ingresaron a Medicina, 3 a Enfermería, 3 a
Tecnología Médica, 3 a Fonoaudiología, 2 a Obstetricia, 2 a Kinesiología y uno a Terapia Ocupacional. Sus puntajes ponderados para el ingreso regular a estas carreras (PAA/PSU Verbal/Lenguaje y Comunicación x 0,25+ Matemáticas x 0,25 + Biología/Ciencias x 0,3 + promedio notas enseñanza media x 0,2 ) se ubicaron por debajo de los puntajes mínimos de ingreso del año correspondiente (Tabla 5). La menor diferencia se observó en un caso admitido a Obstetricia y la mayor en uno que ingresó a Kinesiología.

A diciembre del 2005, 78,6\% de los que ya habían iniciado sus carreras (4 estaban a la espera de comenzar su primer año el 2006) continuaba en la Facultad, cursando desde $1^{\circ}$ a $4^{\circ}$ año. Habían desertado 2 de Medicina ambos por falta de motivación y problemas psicológicos- y 1 de Fonoaudiología había sido excluido por mal rendimiento. Además, un alumno fue transferido desde Medicina a Terapia Ocupacional al final de primer año por la misma última razón. El promedio de notas de la carrera de los estudiantes del Programa fluctuaba entre 3,79 y $5,67(\mathrm{x}=4,92) .84,6 \%$ de ellos $(11 / 13)$ tenía un promedio que se ubicaba en el rango entre -2 DS y +1 DS de la distribución normal del rendimiento de los alumnos regulares de las respectivas carreras. El desempeño no pudo evaluarse en un caso debido a su deserción precoz de la Facultad. Los detalles de la evolución académica de los alumnos dentro de la Facultad se presentarán en una futura publicación.

E1 90,0\% (18/20) de aquellos que no ingresaron a la Facultad tuvo otras posibilidades de educación superior dentro del año siguiente a su salida del Programa. El 75,0\% ingresó a carreras técnicas y el 15,0\% a universitarias.

\section{DISCUSIÓN}

Como parte de las iniciativas chilenas destinadas a generar equidad en la educación universitaria el programa Beca Diego Peralta resulta innovador e integral. En nuestro país, el tema se enfrenta mayoritariamente entregando subsidios económicos -según excelencia 
académica y nivel socio-económico- a quienes ya han rendido la prueba nacional de selección universitaria y se encuentran postulando a una carrera o cursándola. Sin embargo, existe consenso en que la equidad en este ámbito no se logra con sólo asignar recursos para becas, pues ello desconoce la complejidad del problema ${ }^{13}$. Las desigualdades que afectan el éxito en los estudios superiores surgen de una serie de factores personales y sociales que van más allá del plano material. Así entonces, la equidad en la educación superior radica en diversas dimensiones: equidad en las oportunidades de estudio, equidad en el acceso a éstos, equidad en la permanencia y equidad en los resultados ${ }^{14}$. La primera se relaciona con la estructura y oferta del sistema de educación superior. La segunda involucra, entre otros, a los instrumentos de selección. Cabe destacar que las pruebas nacionales de selección universitaria no han contribuido a revertir las desigualdades sociales en el acceso a la educación superior ${ }^{15}$. Sólo un tercio del tramo superior $(20 \%)$ de puntajes PAA pertenecía a los tres quintiles más pobres de la población ${ }^{16}$, lo que no se habría modificado significativamente con la PSU ${ }^{17}$. En la tercera dimensión, equidad en la permanencia, influyen los recursos económicos y otros factores, como las habilidades socioculturales para enfrentar la educación superior, escasas también entre los jóvenes de bajos ingresos ${ }^{13,14}$. La última dimensión se relaciona con la calidad en las instituciones de educación superior.

Llama la atención que no se encontrase ninguna otra iniciativa local que abordara los varios factores generadores de esta inequidad. El programa Beca Diego Peralta es el único que conocemos a nivel nacional que asume como premisa rectora que, para facilitar el ingreso y éxito universitario a los alumnos destacados de escasos recursos, se les debe garantizar tanto las condiciones económicas, como otros apoyos destinados a disminuir las carencias académicas y psicosociales que estos jóvenes tienden además a presentar.

Los resultados del Programa a la fecha muestran que éste generó expectativas y oportunidades de acceso a la educación universitaria que ni los estudiantes ni sus padres imaginaron previamente. A pesar de pertenecer al grupo de los alumnos más destacados de sus respectivos colegios, una proporción muy significativa de ellos y de sus padres no había considerado la universidad como proyecto futuro. Esto podría explicarse en parte, por el escaso acceso real que tienen los jóvenes de nivel socio-económico bajo a ella, pues sólo el $17 \%$ de los universitarios pertenece a los dos quintiles más pobres de la población ${ }^{6}$. Podríamos suponer entonces, que las expectativas de ambos estaban más determinadas por su realidad socioeconómica que por el rendimiento académico de los estudiantes.

La realidad psicosocial de los participantes dificultó también su desempeño en el proceso preingreso y su acceso a la Facultad. Un número considerable presentó psicopatología y/o condiciones de alto riesgo. El fracaso de un tercio (por deserción o exclusión) estuvo determinado en un alto porcentaje por problemas psicológicos. Junto a éstos, cabe destacar el mal rendimiento académico como otro de los factores que se relacionó significativamente con en el fracaso de los alumnos. Es de suponer que en este último factor también pudieron incidir variables psicosociales, tales como el déficit de hábitos y técnicas de estudio y la desorientación vocacional, de alta prevalencia entre los participantes. Lo anterior refuerza la importancia central del apoyo psicosocial en este tipo de programas. Estudios previos han mostrado que la ayuda en este ámbito predice significativa e independientemente el éxito académico en los primeros años de enseñanza superior ${ }^{18,19}$. El paso desde la educación secundaria a la superior sería fuente de mucho estrés, y el apoyo de los alumnos mediante consejería y otras estrategias aumentaría sus posibilidades de éxito ${ }^{19}$. Esta ayuda se consideró de suma importancia en el programa Beca Diego Peralta, cuyos participantes se ven sometidos a un significativo estrés adicional. Este último se vincula, por una parte, a las altas expectativas y exigencias de logro tanto de los alumnos, como de sus familias y las instituciones involucradas. En este sentido, 
cabe destacar que para las familias de estos estudiantes, donde rara vez algún integrante accedió a la educación superior, esta posibilidad resulta un gran hito, lo que puede generar significativa tensión al joven. Por otra parte, se relaciona a la incertidumbre que les produce el hecho de que, aún habiendo sido seleccionados para el Programa, no saben con certeza si finalmente ingresarán a la Facultad. Estos factores pueden además explicar en parte la alta prevalencia observada de psicopatología, pues se trató predominantemente de cuadros reactivos.

El desempeño académico insatisfactorio de algunos pudo también deberse a factores propios del programa. El perfil vocacional y los problemas que presentaron en esta esfera varios de ellos permiten inferir la ausencia de una vocación real por el área de la salud. El éxito de la iniciativa podría incrementarse si se amplía el espectro de carreras ofrecidas.

Los puntajes ponderados de los alumnos finalmente seleccionados para estudiar en la Facultad evidencian que estos jóvenes no habrían ingresado mediante el proceso de admisión regular. Sin embargo, su desempeño a la fecha, ha sido en general satisfactorio. Esto avala la importancia de ampliar los criterios de selección para este tipo de estudiantes si se quiere generar real equidad en el acceso a la educación superior, lo que es refrendado en la literatura internacional ${ }^{20}$. Destaca que Medicina tendió a concentrar los fracasos (3/4), lo que podría atribuirse a su mayor exigencia académica y generación de estrés asociado.

Los datos muestran que el Programa generó oportunidades de desarrollo positivo para gran parte de los participantes y casi la totalidad de aquellos que finalizaron el proceso de apoyo preingreso, principalmente a través de facilitarles el acceso a educación superior (63.2\% y 94.7\%, respectivamente).

Una debilidad del presente análisis es la falta de constancia en el registro de varias de las variables descritas, debida principalmente a la ausencia inicial de un diseño prospectivo de investigación asociado al Programa.

Por último, cabe destacar que no fue posible acceder a ningún programa internacional de características similares. Sin embargo, al menos en Estados Unidos se han llevado a cabo numerosas iniciativas para facilitar el ingreso y retención de alumnos con desventajas socioeconómicas, culturales y étnicas en carreras de la salud. Estos programas se aplican en distintas etapas del proceso educativo (escolar y universitaria) e incluyen diversas estrategias de apoyo (académicas, psicosociales y otras). A la fecha, ha sido difícil comparar sus resultados debido a la falta de indicadores consensuados de éxito y a la inconsistencia de sus métodos de evaluación ${ }^{20-21}$.

Agradecimientos a:

Equipos de apoyo académico, psicosocial, tutoría y secretaría Beca Diego Peralta, Servicio Médico y Dental de los Alumnos Universidad de Chile, Oficina de Comunicaciones, Secretaría de Estudios y Direcciones de Escuelas de la Facultad de Medicina Universidad de Chile, Corporación de Salud y Educación I. Municipalidad de Cerro Navia y Departamento de Salud I. Municipalidad de La Pintana.

\section{REFERENCIAS}

1. BRUNNER JJ., ELACQUA G. Informe Capital Humano en Chile. Santiago: Escuela de Gobierno Universidad Adolfo Ibáñez, 2003. Disponible en: www.uai.cl/p4_home/site/asocfile/ASOCFILE1200 30528134519.pdf. [Consultado el 3 de julio de 2006].

2. FUNDACIÓN PARA LA SUPERACIÓN DE LA POBREZA. Garantías en Educación. En: Moreno L, Rosenblüth M, eds. Umbrales Sociales 2006. Propuesta para la Futura Política Social. Chile: Fundación para la Superación de la Pobreza, 2005: 36-72. Disponible en:www.fundacionpobreza.cl/umbralessociales2006/ cap2.pdf [Consultado el 20 de septiembre de 2006].

3. MINISTERIO DE EDUCACIÓN DE CHILE. GOBIERNO DE CHILE. Reforma Educacional. Capital Humano Avanzado. Disponible en: www.mineduc.cl/index.php?id portal=1\&id seccio $\mathrm{n}=993$ \&id_contenido=939 [Consultado el 3 de julio de 2006].

4. THE WORLD BANK. Education. Data and Statistics. Latin America \& Caribbean (LAC) Countries, 2004. Gross Enrollment Ratio (\%), Tertiary. Disponible en: http://devdata.worldbank.org/edstats/cd2.asp [Consultado el 23 de agosto de 2006].

5. MINISTERIO DE PLANIFICACIÓN, GOBIERNO DE CHILE. Encuesta de Caracterización Socioeconómica Nacional (CASEN) 1990.

6. MINISTERIO DE PLANIFICACIÓN, GOBIERNO DE CHILE. Encuesta de Caracterización Socioeconómica Nacional (CASEN) 2003. 
7. GASPARINI L.Different Lives: Inequality in Latin America and the Caribbean. En: De Ferranti D, Perry G, Ferreira F, Walton M, Coady D, Cunningham W et al, eds. Advance Conference Edition, Inequality in Latin America and the Caribbean: breaking with history? Washington, DC : The International Bank for Reconstruction and Development / The World Bank, 2003 : $35-80$. Disponible en: wbln0018.worldbank.org/LAC/lacinfoclient.nsf/0/3 2d7c0bacee5752a85256dba00545d3f/\$FILE/Inequal ity\%20in\%20Latin\%20America\%20-\%20complete.pdf [Consultado el 23 de agosto de 2006].

8. TRÁMITE FÁCIL.GOBIERNO DE CHILE. Área Educación y Cultura. Becas, Créditos, Subsidios y Fondos Concursables. Disponible en: www.tramitefacil.gov.cl/1481/propertyvalue20865.html [Consultado el 3 de julio 2006].

9. BECAS CHILENAS. UNIVERSIDAD CHILE. Disponible en: http://becas.universia.cl [Consultado el 3 de julio de 2006].

10. RAVEN JC, COURT J, RAVEN J. Matrices Progresivas Escala General. En: Raven JC, Court J, Raven J. Test de Matrices Progresivas. Escalas Coloreada, General y Avanzada. Manual. Buenos Aires: Editorial Paidós, 1993; MPG 1-MPG 37.

11. ANDARIZA I, JIMENEZ N, SEPÚLVEDA M. El Inventario de Preferencias Vocacionales de J. Holland: $\mathrm{Su}$ Introducción en Chile. Memoria para Optar al Título de Psicólogo. Santiago: Pontificia Universidad Católica de Chile, Escuela de Psicología, Departamento de Trabajo y Orientación, 1980.

12. RISSETTI F, MALTES S. Validez Predictiva del MMPI en la Población Universitaria de la Pontificia Universidad Católica de Chile. Pontificia Universidad Católica de Chile, Departamento de Salud Estudiantil. $\mathrm{V}$ Encuentro de Investigación en Admisión Universitaria; 1984 Oct 18-20; Vicuña, Chile.

13. URZUA R. Equidad y Educación Superior: Síntesis de un Debate Reciente. Documento de Trabajo N ${ }^{\circ}$ 149. Santiago: Centro de Análisis de Políticas Públicas, Universidad de Chile, 1999.

14. LEMAITRE MJ. Algunas Precisiones Sobre el Concepto de Equidad y su Abordaje. En: ForoSeminario Equidad y Educación Superior. Santiago: Centro de Análisis de Políticas Públicas, Universidad de Chile, 1998.

15. DONOSO S. La Reforma Educacional y el Sistema de Selección de Alumnos a las Universidades: Impactos y Cambios Demandados. Estudios Pedagógicos 1998; (24):7-30.

16. FUNDACIÓN PARA LA SUPERACIÓN DE LA POBREZA. Brechas Socioeconómicas de la Población Chilena. En: Moreno L, Rosenblüth M, eds. Umbrales Sociales 2006. Propuesta para la Futura Política Social.
Chile: Fundación para la Superación de la Pobreza, $2005: 15-33$. D is pon ible e n : www.fundacionpobreza.cl/umbralessociales2006/ca p1.pdf [Consultado el 22 de agosto de 2006].

17. KOLJATIC M, SILVA M. Improvisación en la Educación: ¿Quién Asume el Costo? Revista Administración y Economía UC 2006; (60). Disponible en: www.ayeuc.cl/Revista60/opinion1 60.htm [Consultado el 21 de agosto de 2006].

18. CUTRONA C, COLE V, COLANGELO N, ASSOULINE S, RUSSELL D. Perceived Parental Social Support and Academic Achievement: an Attachment Theory Perspective. J Pers Soc Psychol 1994; 66:369-378.

19. SCOTT DEBERARD M, SPIELMANS G, JULKA D. Predictors of Academic Achievement and Retention Among College Freshmen: a Longitudinal Study. College Student Journal 2004 Mar. Disponible en: www.highbeam.com/library/docFree.asp?DOCID=1 G1:115034777 [Consultado el 3 de julio de 2006].

20 U.S. DEPARTMENT OF HEALTH AND HUMAN SERVICES. Health Resources and Services Administration COUNCIL ON GRADUATE MEDICAL EDUCATION. Minorities in Medicine: An Ethnic and Cultural Challenge for Physician Training. An Update. Seventeenth Report. April 2005. Disponible en: http://cogme.gov/17thReport/17.pdf [Consultado el 24 de septiembre de 2007].

18 CARLINE J, PATTERSON D, DAVIS L., IRBY D. Precollege Enrichment Programs Intended to Increase the Representation of Minorities in Medicine. Acad Med 1998; 73(3):288-298.

Recepción : 07 jun. 2007 Aprobación : 04 oct. 2007

Usted puede comentar éste y otros artículos publicados en la Revista Chilena de Salud Pública, enviando un correo electrónico a revistasp@med.uchile.cl 